\title{
Recurrent Chronic Lymphocytic Leukemia
}

National Cancer Institute

\section{Source}

National Cancer Institute. Recurrent Chronic Lymphocytic Leukemia. NCI Thesaurus.

Code C8645.

The reemergence of chronic lymphocytic leukemia after a period of remission. 